\title{
ON COMPLETELY INTEGRABLE HAMILTONIAN SYSTEMS PAIRS
}

\section{HAMIDOU DATHE and YATMA MBODJI}

Department of Mathematics and Informatic

Faculty of Sciences and Technics

University Cheikh Anta Diop

Senegal

e-mail: hamidou.dathe@yahoo.fr

yatmambodji87@hotmail.fr

\begin{abstract}
On a differential manifold equipped to symplectic pair $\left(M^{2 h+2 k}, \omega_{1}, \omega_{2}\right)$, we define the notion of completely integrable Hamiltonian system pair then study the problem of this symplectic linearization in a neighbourhood of compact orbit pair.
\end{abstract}

\section{Introduction}

The classic Hamilton's equations are the equations of the flow of a Hamiltonian vector field $X_{H}$ determined by a Hamiltonian function $H$ and a Darboux symplectic form in position and momenta $\omega_{0}=\sum_{i=1}^{n} d p_{i} \wedge d q_{i}$ via the correspondence $i_{X_{H}} \omega_{0}=-d H$. Symplectic geometry generalizes these equations to the general scenario of 2010 Mathematics Subject Classification: 53D15, 53C15, 53D17.

Keywords and phrases: symplectic pairs, Poisson, Hamiltonian system, orbit pair, regular orbit pair, singular orbit pair.

Received October 17, 2017

(C) 2017 Scientific Advances Publishers 
Hamiltonian systems associated to a closed non-degenerate 2 -form(general symplectic form). Among the class of Hamiltonian systems, the sub-class of integrable systems plays a central role. An integrable system on a $2 n$-dimensional symplectic manifold is given by $(n-1)$ additional first integrals $f_{i}$ with the property that each integral (including $H$ ) is preserved by the Hamiltonian flow of the other integrals. This condition is classically known as involutivity of the first integrals and can be written in terms of the Poisson bracket as

$$
\left\{f_{i}, f_{j}\right\}=0 \text {. }
$$

The study of the integrability of such systems is relevant in many areas of mathematics and has its own story. In June 29th of 1853, Joseph Liouville presented a communication entitled "Sur l'intégration des équations différentielles de la Dynamique" at the "Bureau des longitudes". In the resulting note [9] he relates the notion of integrability of the system to the existence of $n$-integrals in involution with respect to the Poisson bracket attached to the symplectic form. A lot of work has been done in the subject after Liouville. Let us outline some of the remarkable achievements from a geometrical and topological point of view. Consider a completely integrable Hamiltonian system. The symplectic gradients of the Hamiltonian function $f_{i}$ define an involutive distribution. Let $\mathcal{O}$ be a regular compact orbit of this distribution then this orbit is a Lagrangian submanifold. Moreover, it is a torus and the neighbouring orbits are also tori. Those tori are called Liouville tori. This is the topological contribution of a theorem which has been known in the literature as Arnold-Liouville theorem. The geometrical contribution of the above-mentioned theorem ensures the existence of symplectic normal forms in the neighbourhood of a compact regular orbit. To the author's knowledge, the works of Henri Mineur [10], [11], [12] already gave a complete description of the Hamiltonian system in a neighbourhood of a compact regular orbit. That is why we will refer to the classical ArnoldLiouville theorem as Liouville-Mineur-Arnold theorem. Let us state the theorem below: Given an completely integrable Hamiltonian system on 
symplectic manifold $\left(M^{2 n}, \omega\right)$, and $\mathcal{O}$ a regular compact orbit. There is a symplectomorphism $\phi$ from a neighbourhood $U(\mathcal{O})$ of $\mathcal{O}$ in $\left(M^{2 n}, \omega\right)$ to $\left(D^{n} \times \mathbb{T}^{n}, \sum_{i=1}^{n} d \mu_{i} \wedge d \beta_{i}\right)$, where $\left(\mu_{i}\right), 1 \leq i \leq n$ is a coordinate on the ball $D^{n}$, and $\left(\beta_{i}\right), 1 \leq i \leq n$ is a periodic coordinate system on the torus $\mathbb{T}^{n}$ such that $\phi^{*} F$ is the map which depend only on the coordinate $\phi^{*}\left(\mu_{i}\right)$. The functions $\phi^{*} \beta_{i}$ are called angle variables and the functions $\phi^{*}\left(\mu_{i}\right)$ are called action variables. The existence of action-angle coordinates in a neighbourhood of a regular compact orbit provides a symplectic model for the Lagrangian foliation $\mathfrak{F}$ determined by the symplectic gradients of the $n$-component functions $f_{i}$ of the moment map $\mu$. In fact, Liouville-Mineur-Arnold theorem entails a uniqueness result for the symplectic structures making $\mathfrak{F}$ into a Lagrangian foliation. In the other words, if $\omega_{1}$ and $\omega_{2}$ are two symplectic structures defined in a neighbourhood of $\mathcal{O}$ for which $\mathfrak{F}$ is Lagrangian then there exists a symplectomorphism preserving the foliation, fixing $\mathcal{O}$ and carrying $\omega_{1}$ to $\omega_{2}$. So if the orbit is regular the existence of action-angle coordinates enables to classify the symplectic germs, up to foliation-preserving symplectomorphism, for which $\mathfrak{F}$ is Lagrangian in a neighbourhood of a compact orbit. There is just one class of symplectic germs for which the foliation is Lagrangian. After this review for symplectic linearisation in a neighbourhood of regular orbit, the following question arises: What can be said about the corresponding classification problem for symplectic germs if the completely integrable systems has singularities? This question is quite natural because singularities are present in many well known examples of integrable systems. In fact, if the completely integrable system is defined on a compact manifold then the singularities cannot be avoided. The symplectic linearisation in a neighbourhood of an singular orbit $\mathcal{O}$ with $\operatorname{dim} \mathcal{O}>0$ is due to Ito in the analytic case [8]. Partial results in the smooth case (with $\operatorname{dim} \mathcal{O}=1$ in a manifold of dimension 4) were obtained by Currás-Bosch and Eva Miranda in [4] and 
independently by Colin de Verdière and San Vu Ngoc in [3]. The final result in any dimension was obtained by Nguyen Tien Zung and Eva Miranda in [13]. In [13], it is also included a $G$-equivariant version of the symplectic linearisation.

In this article, we consider a particular class of manifolds which have been called in the literature symplectic pairs. Symplectic pairs were introduced by Bande and Kotschick in ([2], [1]), where they study the geometry of symplectic pairs. On a such manifolds we define a completely integrable Hamiltonian system pair, then prove an analogue to the symplectic linearisation result which was mentioned above but in the case of completely integrable Hamiltonian systems pairs in manifold equipped to symplectic pair. Precisely, we show that: Given two symplectic pairs $\left(\omega_{1}, \omega_{2}\right)$ and $\left(\omega_{1}^{\prime}, \omega_{2}^{\prime}\right)$ for which the system is a completely integrable Hamiltonian system pair in a neighbourhood of compact orbit $\mathcal{O}$, there is a diffeomorphism preserving the system, fixing $\mathcal{O}$, and sending $\left(\omega_{1}, \omega_{2}\right)$ on $\left(\omega_{1}^{\prime}, \omega_{2}^{\prime}\right)$. In order to prove this result, we will distinguish two situation: the case of regular compact orbit and the case of singular compact orbit.

\section{Preliminaries}

In this section, we recall some basics definitions and properties in symplectic pairs geometry.

\subsection{Hamiltonian vector fields and Poisson bracket}

Definition 2.1. A symplectic pair on a smooth manifold $M^{2 k+2 h}$ of dimension $2 k+2 h$ is a pair of non-trivial closed two-forms $\left(\omega_{1}, \omega_{2}\right)$ such that:

$$
\omega_{1}^{h} \wedge \omega_{2}^{k} \text { is a volume form on } M^{2 k+2 h} \text {, and } \omega_{1}^{k+1}=0, \omega_{2}^{h+1}=0 .
$$

The forms $\omega_{1}$ and $\omega_{2}$ are so necessarily constant class $2 h$ and $2 k$. For $k=0$ or $h=0$, we find the symplectic forms. To a such structure are naturally associated two distributions: the distribution of vector fields 
which annul $\omega_{1}$, and the distribution of vector fields which annul $\omega_{2}$. This distributions are completely integrable because the forms $\omega_{1}$ and $\omega_{2}$ are closed. They determine the characteristic foliations of $\omega_{1}$ and $\omega_{2}$, which we will note $\mathfrak{F}$ and $\mathfrak{G}$.

The characteristic foliation of $\omega_{1}$ is of codimension $2 h$ and her leaves are symplectic manifolds of symplectic structure associated $\omega_{2}$. The characteristic foliation of $\omega_{2}$ is of codimension $2 h$ and her leaves are symplectic manifold of symplectic structure associated $\omega_{1}$.

The foliations $\mathfrak{F}$ and $\mathfrak{G}$ are hollowing out transversal and additional.

Example 2.1. (1) On $\mathbb{R}^{2 k+2 h}$ equipped to coordinates system

$$
\left(x_{1}, \cdots, x_{k}, y_{1}, \cdots, y_{k}, p_{1}, \cdots, p_{h}, q_{1}, \cdots, q_{h}\right) \text { the pair }\left(\omega_{1}, \omega_{2}\right)
$$

defined by:

$$
\omega_{1}=\sum_{i=1}^{h} d x_{i} \wedge d y_{i}, \omega_{2}=\sum_{i=1}^{k} d p_{i} \wedge d q_{i}
$$

is a symplectic pair. This example represent the local model of symplectic pairs.

(2) Let $(M, \omega),\left(M^{\prime}, \omega^{\prime}\right)$ be two symplectic manifolds. Then the produce $M \times M^{\prime}$ equipped to the pair $\left(\omega, \omega^{\prime}\right)$ is a symplectic pair.

Contrary to Riemanian manifolds, the symplectic pairs have not local invariants. This is due to theorem which follows, called the Darboux theorem. It establishes an unique local model of symplectic pairs.

Theorem 2.1 ([1], [2]). Let $\left(\omega_{1}, \omega_{2}\right)$ be a symplectic pair on $M^{2 k+2 h}$ and $p$ be a point of $M^{2 k+2 h}$. Then, it exists a local coordinates system

$$
\left(U, x_{1}, \cdots, x_{k}, y_{1}, \cdots, y_{k}, p_{1}, \cdots, p_{h}, q_{1}, \cdots, q_{h}\right) \text { around of } p \text {, such }
$$

that

$$
\omega_{1 \mid U}=\sum_{i=1}^{k} d x_{i} \wedge d y_{i}, \omega_{2 \mid U}=\sum_{i=1}^{h} d p_{i} \wedge d q_{i}
$$


Every almost symplectic pair $\left(\omega_{1}, \omega_{2}\right)$ on $M^{2 k+2 h}$ induces an isomorphism of $C^{\infty}\left(M^{2 k+2 h}\right)$-modules $b_{\left(\omega_{1}, \omega_{2}\right)}: \chi\left(M^{2 k+2 h}\right) \rightarrow \Omega^{1}\left(M^{2 k+2 h}\right)$ defined by the following proposition:

Proposition 2.1. Let $\left(M^{2 k+2 h}, \omega_{1}, \omega_{2}\right)$ be a symplectic pair. The $\operatorname{map} b_{\left(\omega_{1}, \omega_{2}\right)}: \chi\left(M^{2 k+2 h}\right) \rightarrow \Omega^{1}\left(M^{2 k+2 h}\right)$ defined by:

$$
b_{\left(\omega_{1}, \omega_{2}\right)}(X)=i_{X}\left(\omega_{1}+\omega_{2}\right), \forall X \in \chi\left(M^{2 k+2 h}\right)
$$

is an isomorphism of $C^{\infty}\left(M^{2 k+2 h}, \mathbb{R}\right)$-module.

Proof. Observe that it suffices to show that the map $b_{\left(\omega_{1}, \omega_{2}\right)}$ is injective.

Let $\left(U, x_{1}, \cdots, x_{k}, y_{1}, \cdots, y_{k}, p_{1}, \cdots, p_{h}, q_{1}, \cdots, q_{h}\right)$ be a Darboux coordinate system and $X=\sum_{i=1}^{k}\left(a_{i} \partial x_{i}+b_{i} \partial y_{i}\right)+\sum_{i=1}^{h}\left(c_{i} \partial p_{i}+d_{i} \partial q_{i}\right)$ a vector field on $U$. We assume that $b_{\left(\omega_{1}, \omega_{2}\right)}(X)=0$. Then we have

$$
\begin{aligned}
i_{X}\left(\omega_{1}+\omega_{2}\right)(X) & =0 \\
i_{X} \omega_{1} & =-i_{X} \omega_{2} .
\end{aligned}
$$

Consequently,

$$
\begin{aligned}
i_{X}\left(\omega_{1}\right)\left(\partial x_{i}\right) & =i_{X} \omega_{2}\left(\partial x_{i}\right) \\
i_{X}\left(\omega_{1}\right)\left(\partial x_{i}\right) & =0 \\
b_{i} & =0, \\
i_{X}\left(\omega_{1}\right)\left(\partial y_{i}\right) & =i_{X} \omega_{2}\left(\partial y_{i}\right) \\
i_{X}\left(\omega_{1}\right)\left(\partial y_{i}\right) & =0 \\
a_{i} & =0,
\end{aligned}
$$




$$
\begin{aligned}
i_{X}\left(\omega_{1}\right)\left(\partial p_{i}\right) & =i_{X} \omega_{2}\left(\partial p_{i}\right) \\
i_{X}\left(\omega_{2}\right)\left(\partial p_{i}\right) & =0 \\
d_{i} & =0, \\
i_{X}\left(\omega_{1}\right)\left(\partial q_{i}\right) & =i_{X} \omega_{2}\left(\partial q_{i}\right) \\
i_{X}\left(\omega_{2}\right)\left(\partial q_{i}\right) & =0 \\
c_{i} & =0 .
\end{aligned}
$$

According to relations (1), (2), (3), and (4), we deduce that $X=0$. Thus the map $b_{\left(\omega_{1}, \omega_{2}\right)}$ is injective.

Thanks to this isomorphism, we can associate at every function $f \in C^{\infty}\left(M^{2 k+2 h}, \mathbb{R}\right)$ an unique vectors field $X_{f} \in \chi(M)$, called Hamiltonian vector field of $f$.

Definition 2.2. Let $\left(M^{2 k+2 h}, \omega_{1}, \omega_{2}\right)$ be a symplectic pair and $f \in \mathrm{C}^{\infty}(M, \mathbb{R})$ a function is called Hamiltonian vectors field of $f$ the single vectors field $X_{f}$ defined by

$$
X_{f}=b_{\left(\omega_{1}, \omega_{2}\right)}^{-1}(-d f)
$$

Likewise,

$$
i_{X_{f}}\left(\omega_{1}+\omega_{2}\right)=-d f
$$

The Hamiltonian vector field verify the following properties:

Property 2.1. For all f and $g \in \mathrm{C}^{\infty}\left(M^{2 n+1}, \mathbb{R}\right)$, we have:

(1) $X_{f+g}=X_{f}+X_{g}$.

(2) $X_{f g}=f X_{g}+g X_{f}$. 
(3) If the Hamiltonian functions $f$ and $g$ are associated to the same Hamiltonian vector field $X$, then the function $(f-g)$ is locally constant.

(4) If $X$ is a Hamiltonian vector field associated to $f, Y$ the component of $X$ tangent to $\mathfrak{F}$ and $Z$ his component tangent to $\mathfrak{G}$, then $Y$ and $Z$ verify the equations

$$
i_{Y}\left(\omega_{1}+\omega_{2}\right)=-\frac{1}{2} d f, i_{Z}\left(\omega_{1}+\omega_{2}\right)=-\frac{1}{2} d f
$$

Proof. Let $f, g \in \mathrm{C}^{\infty}\left(M^{2 n+1}, \mathbb{R}\right)$. For the first property, we have

$$
\begin{aligned}
i_{X_{f+g}\left(\omega_{1}+\omega_{2}\right)}=-d(f+g) \\
=-d f-d g \\
=i_{X_{f}}\left(\omega_{1}+\omega_{2}\right)+i_{X_{g}}\left(\omega_{1}+\omega_{2}\right) \\
=i_{X_{f}+X_{g}}\left(\omega_{1}+\omega_{2}\right) \\
b_{\left(\omega_{1}, \omega_{2}\right)}\left(X_{f+g}\right)=b_{\left(\omega_{1}, \omega_{2}\right)}\left(X_{f}+X_{g}\right) .
\end{aligned}
$$

Since the map $b_{\left(\omega_{1}, \omega_{2}\right)}$ is an isomorphism, then according to relation (6), we obtain $X_{f+g}=X_{f}+X_{g}$. For the second property, we have

$$
\begin{aligned}
i_{X_{f g}}\left(\omega_{1}+\omega_{2}\right) & =-d(f g) \\
& =-g d f-f d g \\
& =g i_{X_{f}}\left(\omega_{1}+\omega_{2}\right)+f i_{X_{g}}\left(\omega_{1}+\omega_{2}\right) \\
& =i_{g X_{f}+f X_{g}}\left(\omega_{1}+\omega_{2}\right) \\
b_{\left(\omega_{1}, \omega_{2}\right)}\left(X_{f g}\right) & =b_{\left(\omega_{1}, \omega_{2}\right)}\left(g X_{f}+f X_{g}\right) .
\end{aligned}
$$

Since the map $b_{\left(\omega_{1}, \omega_{2}\right)}$ is an isomorphism, then according to relation (7), we have $X_{f g}=g X_{f}+f X_{g}$. For the thirty property, we have 


$$
\begin{aligned}
d(f-g) & =d f-d g \\
& =i_{X}\left(\omega_{1}+\omega_{2}\right)-i_{X}\left(\omega_{1}+\omega_{2}\right) \\
& =0 .
\end{aligned}
$$

For the last property, let $X_{1}$ and $X_{2}$ be two vector fields tangent to $\mathfrak{F}$ and $\mathfrak{G}$, respectively. We assume that $i_{X_{1}}\left(\omega_{1}+\omega_{2}\right)=-\frac{1}{2} d f, i_{X_{2}}\left(\omega_{1}+\omega_{2}\right)=-\frac{1}{2} d f$. We have

$$
\begin{gathered}
i_{Y+Z}\left(\omega_{1}+\omega_{2}\right)=i_{X_{1}+X_{2}}\left(\omega_{1}+\omega_{2}\right) \\
i_{Y-X_{1} \omega_{2}}=i_{-Z+X_{2}} \omega_{1} .
\end{gathered}
$$

Since $\omega_{2}$ is non degenerated on $\mathfrak{F}$ and $\omega_{1}$ is non degenerated on $\mathfrak{G}$, then according to relation (8), we obtain $Y=X_{1}$ and $Z=X_{2}$.

The proposition that follows, show that it exists a Poisson bracket $\{$,$\} on C^{\infty}\left(M^{2 k+2 h}, \mathbb{R}\right)$ such that the map $\left(C^{\infty}\left(M^{2 k+2 h}, \mathbb{R}\right)\right.$, $\{\},) \rightarrow\left(\chi\left(M^{2 k+2 h}\right),[],\right)$ defined by $f \rightarrow X_{f}$ is a Lie algebra antihomomorphism with respect to the Poisson bracket and the commutator of vector fields.

Proposition 2.2. It exists a Lie algebra structure $\{$,$\} on C^{\infty}\left(M^{2 k+2 h}, \mathbb{R}\right)$ such that for all $f, g \in C^{\infty}\left(M^{2 k+2 h}, \mathbb{R}\right)$, we have

$$
X_{\{f, g\}}=-\left[X_{f}, X_{g}\right]
$$

Proof. We put

$$
\{f, g\}=\left(\omega_{1}+\omega_{2}\right)\left(X_{f}, X_{g}\right)
$$

Since $\omega_{1}$ and $\omega_{2}$ are bilinear and antisymmetric, then $\{$,$\} is bilinear$ and antisymmetric. 
For Leibniz identity, we consider $f, g$ and $h \in \mathrm{C}^{\infty}$. We have

$$
\begin{aligned}
\{f, g h\} & =\left(\omega_{1}+\omega_{2}\right)\left(X_{f}, X_{g h}\right) \\
& =\left(\omega_{1}+\omega_{2}\right)\left(X_{f}, g X_{h}+h X_{g}\right) \\
& =\left(\omega_{1}+\omega_{2}\right)\left(X_{f}, g X_{h}\right)+\left(\omega_{1}+\omega_{2}\right)\left(X_{f}, h X_{g}\right) \\
& =g\left(\omega_{1}+\omega_{2}\right)\left(X_{f}, X_{h}\right)+h\left(\omega_{1}+\omega_{2}\right)\left(X_{f}, X_{g}\right) \\
& =g\{f, h\}+h\{f, g\} .
\end{aligned}
$$

For Jacobi identity, we consider $f, g$ and $h \in \mathrm{C}^{\infty}\left(M^{2 k+2 h}\right)$. For all $f \in \mathrm{C}^{\infty}\left(M^{2 k+2 h}, \mathbb{R}\right)$, the equation

$$
i_{Y}\left(\omega_{1}+\omega_{2}\right)=-\frac{1}{2} d f
$$

has a unique well-defined solution when restricted to $\left(\mathfrak{F}, \omega_{1}\right)$ and to $\left(\mathfrak{G}, \omega_{2}\right)$. We denote by $Y_{f}$ and $Z_{f}$ the Hamiltonian vector fields of function $\frac{1}{2} f$ with respect to the symplectic structure $\omega_{1}$ on $\mathfrak{F}$ and the symplectic structure $\omega_{2}$ on $\mathfrak{G}$, respectively. According to relation (5), we can write

$$
X_{f}=Y_{f}+Z_{f}
$$

Thus, we obtain

$$
\begin{aligned}
\{f,\{g, h\}\} & =\left(\omega_{1}+\omega_{2}\right)\left(Y_{f}+Z_{f}, Y_{\{g, h\}}+Z_{\{g, h\}}\right) \\
& =\omega_{1}\left(Y_{f}, Y_{\{g, h\}}\right)+\omega_{2}\left(Z_{f}, Z_{\{g, h\}}\right) .
\end{aligned}
$$

Since $\omega_{1}$ is a symplectic form adapted to $\mathfrak{F}$ and $\omega_{2}$ the symplectic form adapted to $\mathfrak{G}$, then we have

$$
\omega_{1}\left(Y_{f}, Y_{\{g, h\}}\right)=-\omega_{1}\left(Y_{g}, Y_{\{h, f\}}\right)-\omega_{1}\left(Y_{h}, Y_{\{f, g\}}\right),
$$


and

$$
\omega_{2}\left(Z_{f}, Z_{\{g, h\}}\right)=-\omega_{2}\left(Z_{g}, Z_{\{h, f\}}\right)-\omega_{2}\left(Z_{h}, Z_{\{f, g\}}\right)
$$

According to relation we obtain (2) and (3)

$$
\begin{aligned}
\{f,\{g, h\}\}= & -\left(\omega_{1}+\omega_{2}\right)\left(Y_{g}+Z_{g}, Y_{\{h, f\}}+Z_{\{h, f\}}\right) \\
& -\left(\omega_{1}+\omega_{2}\right)\left(Y_{h}+Z_{h}, Y_{\{f, g\}}+Z_{\{f, g\}}\right) \\
= & -\{g,\{h, f\}\}-\{h,\{f, g\}\} .
\end{aligned}
$$

Let $f, g$ be a smooth functions, we have

$$
\begin{aligned}
i_{\left[X_{f}, X_{g}\right]}\left(\omega_{1}+\omega_{2}\right) & =L_{X_{f}} i_{X_{g}}\left(\omega_{1}+\omega_{2}\right)-i_{X_{g}} L_{X_{f}}\left(\omega_{1}+\omega_{2}\right) \\
& =L_{X_{f}}(-d g)-i_{X_{g}} d\left(i_{X_{f}}\left(\omega_{1}+\omega_{2}\right)\right) \\
& =d i_{X_{f}}(-d g)-i_{X_{g}} d(-d f) \\
& =-d\left(d g\left(X_{f}\right)\right) \\
& =d\{f, g\} .
\end{aligned}
$$

Thus we obtain

$$
X_{\{f, g\}}=-\left[X_{f}, X_{g}\right]
$$

By elementary calculus, we obtain the following proposition:

Proposition 2.3. Let $\left(U, x_{1}, \cdots, x_{k}, y_{1}, \cdots, y_{k}, p_{1}, \cdots, p_{h}, q_{1}, \cdots, q_{h}\right)$ be a Darboux coordinates system on $\left(M^{2 k+2 h}, \omega_{1}, \omega_{2}\right)$ and $f \in C^{\infty}\left(M^{2 k+2 h+1}, \mathbb{R}\right)$. So we have

$$
\begin{gathered}
X_{f \mid U}=-\frac{\partial f}{\partial y_{i}} \partial_{x_{i}}+\frac{\partial f}{\partial x_{i}} \partial_{y_{i}}-\frac{\partial f}{\partial q_{i}} \partial_{p_{i}}+\frac{\partial f}{\partial p_{i}} \partial_{q_{i}}, \\
\{f, g\}_{\mid U}=\left(\frac{\partial f}{\partial x_{i}} \frac{\partial g}{\partial y_{i}}-\frac{\partial f}{\partial y_{i}} \frac{\partial g}{\partial x_{i}}\right)+\left(\frac{\partial f}{\partial p_{i}} \frac{\partial g}{\partial q_{i}}-\frac{\partial f}{\partial q_{i}} \frac{\partial g}{\partial p_{i}}\right) .
\end{gathered}
$$


In particular,

$$
\begin{gathered}
X_{x_{i}}=\partial y_{i} ; X_{y_{i}}=-\partial x_{i}, \\
X_{p_{i}}=\partial q_{i} ; X_{q_{i}}=\partial p_{i}, \\
\left\{x_{i}, y_{j}\right\}=\delta_{i j} ;\left\{p_{i}, q_{i}\right\}=\delta_{i j}, \\
\left\{x_{i}, p_{j}\right\}=0 ;\left\{p_{i}, y_{i}\right\}=0, \\
\left\{x_{i}, q_{j}\right\}=0 ;\left\{y_{i}, q_{i}\right\}=0 .
\end{gathered}
$$

Let us recall the notion of automorphism.

Definition 2.3. Let $\left(M, \omega_{1}, \omega_{2}\right),\left(M^{\prime}, \omega_{1}^{\prime}, \omega_{2}^{\prime}\right)$, be two symplectic pairs. A diffeomorphism $\phi: M \rightarrow M^{\prime}$ is called an automorphism, if

$$
\left\{\begin{array}{l}
\phi^{\star}\left(\omega_{1}^{\prime}\right)=\omega_{1}, \\
\phi^{\star}\left(\omega_{2}^{\prime}\right)=\omega_{2} .
\end{array}\right.
$$

\subsection{Completely integrable Hamiltonian systems pairs and}

\section{Lagrangian foliations pairs}

Definition 2.4. Let $\left(M^{2 k+2 h}, \omega_{1}, \omega_{2}\right)$ be a symplectic pair and $f_{1}, \cdots, f_{h}, g_{1} \cdots, g_{k}$ be $(k+h)$-Hamiltonian functions on $\left(M^{2 k+2 h}, \omega_{1}, \omega_{2}\right)$. We said that $\left(f_{1}, \cdots, f_{k}, g_{1}, \cdots, g_{h}\right)$ is a completely integrable Hamiltonian system pair if the following conditions are verified:

- The Hamiltonian vector fields $X_{f_{i}}$, are tangent to $\mathfrak{F}$ and the Hamiltonian vector fields $X_{g_{i}}$ are tangent to $\mathfrak{G}$.

- $\left\{f_{i}, f_{j}\right\}=\left\{g_{i}, g_{j}\right\}=\left\{f_{i}, g_{j}\right\}=0$ for all $i, j$.

- The system $\left(d f_{1}, \cdots, d f_{k}, d g_{1}, \cdots, d g_{h}\right)$ is linearly independent almost everywhere. 
The functions $f_{i}, g_{i}$ are called first integrals of the integrable system. Given a completely integrable Hamiltonian system pair, there are a local Hamiltonian $\mathbb{R}^{k+h}$-action of momentum map $\mu=\left(f_{1}, \cdots, f_{k}\right.$, $\left.g_{1}, \cdots, g_{h}\right)$ and two foliations naturally attached to it.

Proposition 2.4. Let $\left(f_{1}, \cdots, f_{k}, g_{1}, \cdots, g_{h}\right)$ be a completely integrable Hamiltonian system pair on symplectic pair $\left(M^{2 k+2 h}, \omega_{1}, \omega_{2}\right)$. Assume that $p \in M^{2 k+2 h}$ is a point for which $d_{p} f_{1} \cdots \wedge d_{p} f_{k} \wedge d g_{1} \cdots \wedge d g_{h} \neq 0$. Then the distributions $\mathcal{D}_{1}=<X_{f_{1}}, \cdots, X_{f_{k}}>, \mathcal{D}_{2}=<X_{g_{1}}, \cdots, X_{g_{h}}>$ are involutive and the tangent spaces at $p$ to leaves through $p$ are respectively, a Lagrangian subspace of $\left(\operatorname{ker} \omega_{2}(p), \omega_{1}(p)\right)$ and $\left(\operatorname{ker} \omega_{1}(p), \omega_{2}(p)\right)$.

Proof. On the one hand, since $\left[X_{f_{i}} ; X_{f_{j}}\right]=X_{\left\{f_{i}, f_{j}\right\}}$, the condition $\left\{f_{i}, f_{j}\right\}=0$ implies $\left[X_{f_{i}}, X_{f_{j}}\right]=0$ for all $i, j$ and the distribution $\mathcal{D}_{1}$ is involutive. On the other hand, since $\left[X_{g_{i}} ; X_{g_{j}}\right]=X_{\left\{g_{i}, g_{j}\right\}}$, the condition $\left\{g_{i}, g_{j}\right\}=0$ implies $\left[X_{g_{i}}, X_{g_{j}}\right]=0$ for all $i, j$ and the distribution $\mathcal{D}_{2}$ is also involutive. Let $\mathcal{F}$ and $\mathcal{G}$ be a leaves through at $p$ of distributions $\mathcal{D}_{1}$ and $\mathcal{D}_{2}$, respectively. From the definition of Poisson bracket $\left\{f_{i}, f_{j}\right\}=\omega\left(X_{f_{i}}, X_{f_{j}}\right)$ and $\left\{g_{i}, g_{j}\right\}=\omega\left(X_{g_{i}}, X_{g_{j}}\right)$, the tangent spaces $T_{p} \mathcal{F}$ and $T_{p} \mathcal{G}$ are isotropic. The condition $d_{p} f_{1} \wedge \cdots \wedge d_{p} f_{k} \wedge$ $d_{p} g_{1} \wedge \cdots \wedge d_{p} g_{h} \neq 0$ implies that the Hamiltonian vector fields $X_{f_{i}}$ span an $k$-dimensional vector space at the point $p$ and the Hamiltonian vector fields $X_{g_{i}}$ span an $h$-dimensional vector space at the point $p$. Therefore, the tangent space at $p$ of the leaf $\mathcal{F}$ is Lagrangian subspace to $\left(\right.$ ker $\left.\omega_{2}(p), \omega_{1}(p)\right)$ and the tangent space at $p$ of the leaf $\mathcal{G}$ is Lagrangian subspace to $\left(\operatorname{ker} \omega_{1}(p), \omega_{2}(p)\right)$. 
In all we note $\mathfrak{F}_{1}$ the foliation defined by $\mathcal{D}_{1}$ and $\mathfrak{F}_{2}$ the foliation defined by $\mathcal{D}_{2}$. The pair $\left(\mathfrak{F}_{1}, \mathfrak{F}_{2}\right)$ is called the Lagrangian foliation pair attached to integrable Hamiltonian system pair.

Example 2.2. (1) On $\mathbb{R}^{2 k+2 h}$ equipped to standard symplectic pair

$$
\omega_{2}=\sum_{i=1}^{h} d p_{i} \wedge d q_{i}, \omega_{1}=\sum_{i=1}^{k} d x_{i} \wedge d y_{i}
$$

The system $f_{1}=x_{1}^{2}+y_{1}^{2}, \cdots, f_{k}=x_{k}^{2}+y_{k}^{2}, g_{1}=p_{1}^{2}+q_{1}^{2}, \cdots, g_{h}=p_{h}^{2}+q_{h}^{2}$ is a completely integrable Hamiltonian system pair. The foliation $\mathfrak{F}_{1}$ is generated by vector fields $X_{i}=2\left(-y_{i} \partial x_{i}+x_{i} \partial y_{i}\right)$ for $1 \leq i \leq k$ and the foliation $\mathfrak{F}_{2}$ is generated by vector fields $X_{i}=2\left(-q_{i} \partial p_{i}+p_{i} \partial q_{i}\right)$ for $1 \leq i \leq h$.

(2) On $\mathbb{T}^{2} \times \mathbb{S}^{2} \times \mathbb{S}^{1}$ equipped to symplectic pair

$$
\omega_{1}=d \alpha \wedge d \phi, \omega_{2}=d \beta \wedge d \theta .
$$

The system $f_{1}=\alpha \phi, f_{2}=\beta \theta$ is a completely integrable Hamiltonian pair. The foliation $\mathfrak{F}$ is generated by $X_{1}=-\alpha \partial \alpha+\phi \partial \phi, X_{2}=-\beta \partial \beta+\theta \partial \theta$.

\subsection{Regular orbits pairs and singular orbits pairs}

Let $\left(f_{1}, \cdots, f_{k}, g_{1}, \cdots, g_{h}\right)$ be a completely integrable Hamiltonian system pair of momentum map $\mu$ on symplectic pair $\left(M^{2 k+2 h}, \omega_{1}, \omega_{2}\right)$, $p$ be a point in $M^{2 k+2 h}, \mathcal{O}_{1}$ be the orbit of $\mathcal{D}_{1}$ through $p$, and $\mathcal{O}_{2}$ be the orbit of $\mathcal{D}_{2}$ through $p$. We denote by $\pi_{1}: \mathbb{R}^{k+h} \rightarrow \mathbb{R}^{k}$ and $\pi_{2}: \mathbb{R}^{k+h} \rightarrow \mathbb{R}^{h}$ the canonical projections.

Definition 2.5. We said that $\left(\mathcal{O}_{1}, \mathcal{O}_{2}\right)$ is a regular orbit pair if $\operatorname{rank}\left(d_{p} \pi_{1} \circ \mu\right)=k$ and $\operatorname{rank}\left(d_{p} \pi_{2} \circ \mu\right)=h$.

Remark 2.1. If a point in orbit pair $\left(\mathcal{O}_{1}, \mathcal{O}_{2}\right)$ is regular then all point in $\left(\mathcal{O}_{1}, \mathcal{O}_{2}\right)$ is regular. Because regularity is a property which is invariant under the local Hamiltonian $\mathbb{R}^{k+h}$-action. 
Definition 2.6. We said that $\left(\mathcal{O}_{1}, \mathcal{O}_{2}\right)$ is singular orbit pair of rang $(r, s)$ if $\operatorname{rank}\left(d_{p} \pi_{1} \circ \mu\right)=r$ and $\operatorname{rank}\left(d_{p} \pi_{2} \circ \mu\right)=s$.

Remark 2.2. If a point in orbit pair $\left(\mathcal{O}_{1}, \mathcal{O}_{2}\right)$ is singular orbit pair of rank $(r, s)$ then all point in $\left(\mathcal{O}_{1}, \mathcal{O}_{2}\right)$ is singular of rank $(r, s)$. Because singularity is a property which is invariant under the local Hamiltonian $\mathbb{R}^{k+h}$-action.

In the following section, we study the symplectic pair linearisation problem in a neighbourhood of regular compact orbit pair $\left(\mathcal{O}_{1}, \mathcal{O}_{2}\right)$.

\section{Symplectic Pair Linearisation in a Neighbourhood of Regular Compact Orbit Pair}

Consider a symplectic pair $\left(M^{2 k+2 h}, \omega_{1}, \omega_{2}\right)$ equipped a completely integrable Hamiltonian system pair $\left(f_{i}, \cdots, f_{k}, g_{1}, \cdots, g_{h}\right)$ and $\left(\mathcal{O}_{1}, \mathcal{O}_{2}\right)$ a regular compact orbit pair. In this section, we want to prove that under the above assumptions, there exist coordinates in a neighbourhood of $\left(\mathcal{O}_{1}, \mathcal{O}_{2}\right)$ such that the foliation can be linearised.

Theorem 3.1. There is an automorphism $\phi$ from a neighbourhood $\left(U\left(\mathcal{O}_{1}, \mathcal{O}_{2}\right), \omega_{1}, \omega_{2}\right)$ of $\left(\mathcal{O}_{1}, \mathcal{O}_{2}\right)$ in $\left(M^{2 k+2 h}, \omega_{1}, \omega_{2}\right)$ to $\left(\mathbb{D}^{k} \times \mathbb{T}^{k} \times \mathbb{D}^{h} \times \mathbb{T}^{h}\right.$, $\left.\omega_{1}=\sum_{i=1}^{k} d x_{i} \wedge d y_{i}, \omega_{2}=\sum_{i=1}^{n} d p_{i} \wedge d q_{i}\right)$, where $\left(x_{1}, \cdots, x_{k}\right)$ is a coordinate system on a ball $\mathbb{D}^{k},\left(y_{1}, \cdots, y_{k}\right)$ is a periodic coordinate system on the torus $\mathbb{T}^{k},\left(p_{1}, \cdots, p_{h}\right)$ is a coordinate system on a ball $\mathbb{D}^{h}$, and $\left(q_{1}, \cdots, q_{h}\right)$ is a periodic coordinate system on the torus $\mathbb{T}^{h}$ such that $\phi^{*} \mu$ is a map which depends only on the variables $x_{1}, \cdots, x_{k}, p_{1}, \cdots, p_{h}$. The functions $\phi^{*} y_{i}, \phi^{*} q_{i}$ on $U\left(\mathcal{O}_{1}, \mathcal{O}_{2}\right)$ are called angle variables, the functions $\phi^{*} x_{i}, \phi^{*} p_{i}$ on $U\left(\mathcal{O}_{1}, \mathcal{O}_{2}\right)$ are called action variables. 
Proof. Since the orbits $\mathcal{O}_{1}, \mathcal{O}_{2}$ are compact then the produce $\mathcal{O}_{1} \times \mathcal{O}_{2}$ is a closed of $M^{2 k+2 h}$. The manifold $M^{2 k+2 h}$ being normal, then it exist a local coordinate system $\left(U, \alpha_{1}, \cdots, \alpha_{k}, \beta_{1}, \cdots, \beta_{k}, \theta_{1}, \cdots, \theta_{h}\right.$, $\left.\mu_{1}, \cdots, \mu_{h}\right)$ around $\left(\mathcal{O}_{1} \times \mathcal{O}_{2}\right)$ in $M^{2 k+2 h}$ such that

$$
U \cap\left(\mathcal{O}_{1} \times \mathcal{O}_{2}\right)=\mathcal{O}_{1} \times \mathcal{O}_{2}
$$

Let $N_{1}, N_{2}$ be the submanifolds defined by $N_{1}:\left\{\theta_{1}=\cdots=\theta_{h}=\mu_{1}=\right.$ $\left.\cdots=\mu_{h}=0\right\}$ and $N_{2}:\left\{\alpha_{1}=\cdots=\alpha_{k}=\beta_{1}=\cdots=\beta_{k}=0\right\}$. Observe that $\omega_{1}$ and $\omega_{2}$ are respectively, a symplectic forms on $N_{1}$ and $N_{2}$. Thus, the equations

$$
i_{X} \omega_{1}=-d f_{i} \text { and } i_{X} \omega_{2}=-d g_{i}
$$

have a unique well-defined solution when restricted to the symplectic submanifolds $\left(N_{1}, \omega_{1}\right)$ and $\left(N_{2}, \omega_{2}\right)$, respectively. We denote, respectively by $Y_{f_{i}}$ and $Z_{f_{i}}$ the solution of this equations. With all these information at hand we can write $X_{f_{i}}=Y_{f_{i}}$ and $X_{g_{i}}=Z_{f_{i}}$, where $X_{f_{i}}$ and $X_{g_{i}}$ are the Hamiltonian vector fields with respect to symplectic pair $\left(\omega_{1}, \omega_{2}\right)$. Observe that, the Hamiltonian vector fields $Y_{f_{1}}, \cdots, Y_{f_{k}}$ define a completely integrable Hamiltonian system on $\left(N_{1}, \omega_{1}\right)$ and the Hamiltonian vector fields $Z_{g_{1}}, \cdots, Z_{g_{h}}$ define a completely integrable Hamiltonian system on $\left(N_{2}, \omega_{2}\right)$. Indeed, we have

$$
\begin{aligned}
\left\{f_{i}, f_{j}\right\}_{N_{1}} & =\omega_{1}\left(Y_{f_{i}}, Y_{f_{j}}\right) \\
& =\left(\omega_{1}+\omega_{2}\right)\left(X_{f_{i}}, X_{f_{j}}\right) \\
& =\left\{f_{i}, f_{j}\right\} \\
& =0,
\end{aligned}
$$


and

$$
\begin{aligned}
\left\{g_{i}, g_{j}\right\}_{N_{2}} & =\omega_{2}\left(Z_{g_{i}}, Z_{g_{j}}\right) \\
& =\left(\omega_{1}+\omega_{2}\right)\left(X_{g_{i}}, X_{g_{j}}\right) \\
& =\left\{g_{i}, g_{j}\right\} \\
& =0 .
\end{aligned}
$$

Moreover, $\mathcal{O}_{1}$ is a regular compact orbit of integrable Hamiltonian system $Y_{f_{1}}, \cdots, Y_{f_{k}}$ in $\left(N_{1}, \omega_{1}\right)$ and $\mathcal{O}_{2}$ is a regular compact orbit of integrable Hamiltonian system $Z_{f_{1}}, \cdots, Z_{f_{h}}$ in $\left(N_{2}, \omega_{2}\right)$. According to Liouville-Mineur-Arnold theorem (see [10], [11], [12]), there is a symplectomorphism $\phi_{1}$ from a neighbourhood $U_{1}\left(\mathcal{O}_{1}\right)$ of $\mathcal{O}_{1}$ in $\left(N_{1}, \omega_{1}\right)$ to $\left(\mathrm{D}^{k} \times \mathbb{T}^{k}, \sum_{i=1}^{k} d \alpha_{i} \wedge d \beta_{i}\right)$, where $\left(\alpha_{i}\right), 1 \leq i \leq k$ is a coordinate on the ball $D^{k}$, and $\left(\beta_{i}\right), 1 \leq i \leq k$ is a periodic coordinate system on the torus $\mathbb{T}^{k}$ such that $\phi_{1}^{*}\left(\pi_{1} \circ \mu\right)$ is the map which depend only on the coordinate $\phi_{1}^{*}\left(\alpha_{i}\right)$, and a symplectomorphism $\phi_{2}$ from a neighbourhood $U_{2}\left(\mathcal{O}_{2}\right)$ of $\mathcal{O}_{2} \quad$ in $\left(N_{2}, \omega_{2}\right)$ to $\left(D^{h} \times \mathbb{T}^{h}, \sum_{i=1}^{h} d \theta_{i} \wedge d \mu_{i}\right)$, where $\left(\theta_{i}\right), 1 \leq i \leq h$ is a coordinate on the ball $D^{h}$, and $\left(\theta_{i}\right), 1 \leq i \leq h$ is a periodic coordinate system on the torus $\mathbb{T}^{h}$ such that $\phi_{2}^{*}\left(\pi_{2} \circ \mu\right)$ is the map which depend only on the coordinate $\phi_{2}^{*}\left(\theta_{i}\right)$. Now, we define the map $\phi: U_{1}\left(\mathcal{O}_{1}\right) \times U_{2}\left(\mathcal{O}_{2}\right) \rightarrow \times \mathbb{D}^{k} \times \mathbb{T}^{k} \times \mathbb{D}^{h} \times \mathbb{T}^{h}$ by

$$
\phi\left(z_{1}, z_{2}\right)=\left(\phi_{1}\left(z_{1}\right), \phi_{2}\left(z_{2}\right)\right), \text { for all }\left(z_{1}, z_{2}\right) \in U_{1}\left(\mathcal{O}_{1}\right) \times U_{2}\left(\mathcal{O}_{2}\right) \text {. }
$$

This map is an automorphism. Indeed, we have

$$
\begin{aligned}
\phi^{*}\left(\sum_{i=1}^{n} d \alpha_{i} \wedge d \beta_{i}\right) & =\phi_{1}^{*}\left(\sum_{i=1}^{n} d \alpha_{i} \wedge d \beta_{i}\right) \\
& =\omega_{1},
\end{aligned}
$$


and

$$
\begin{aligned}
\phi^{*}\left(\sum_{i=1}^{n} d \theta_{i} \wedge d \mu_{i}\right) & =\phi_{2}^{*}\left(\sum_{i=1}^{n} d \alpha_{i} \wedge d \beta_{i}\right) \\
& =\omega_{2} .
\end{aligned}
$$

Moreover, the map $\phi^{*} \mu$ depend only on variables $\phi^{*}\left(\alpha_{i}\right)$ and $\phi^{*}\left(\theta_{i}\right)$. This ends the proof of the theorem.

This theorem enables to classify the symplectic pair germs, up to foliation-preserving automorphism, for which the system $\left(f_{1}, \cdots, f_{k}\right.$, $\left.g_{1}, \cdots, f_{h}\right)$ is a completely integrable Hamiltonian system pair in a neighbourhood of a regular compact orbit pair. There is just one class of symplectic pair germs for which the system $\left(f_{1}, \cdots, f_{k}, g_{1}, \cdots, g_{h}\right)$, is a completely integrable Hamiltonian system pair.

Theorem 3.2. If $\left(\omega_{1}^{\prime}, \omega_{2}^{\prime}\right)$ is another symplectic pair for which the system $\left(f_{1}, \cdots, f_{k}, g_{1}, \cdots, g_{h}\right)$, is a completely integrable Hamiltonian system pair, then there exists a diffeomorphism $\phi$ defined in a neighbourhood of $\left(\mathcal{O}_{1}, \mathcal{O}_{2}\right)$ such that:

- It fix the orbit pair $\left(\mathcal{O}_{1}, \mathcal{O}_{2}\right)$.

- It preserve the foliation pair $\left(\mathfrak{F}_{1}, \mathfrak{F}_{2}\right)$.

- $\phi^{*}\left(\omega_{1}^{\prime}\right)=\omega_{1}, \phi^{*}\left(\omega_{2}^{\prime}\right)=\omega_{2}$.

In this case we said that the symplectic pair are equivalent, and we note $\left(\omega_{1}, \omega_{2}\right) \sim_{\left(\mathfrak{F}_{1}, \mathfrak{F}_{2}\right)}\left(\omega_{1}^{\prime}, \omega_{2}^{\prime}\right)$. 


\section{Symplectic Pair Linearisation in a Neighbourhood of Singular Compact Orbit}

In all that follows, $\left(M^{2 k+2 h}, \omega_{1}, \omega_{2}\right)$ designate a symplectic pair, $\left(f_{1}, \cdots, f_{k}, g_{1}, \cdots, g_{h}\right)$, a completely integrable Hamiltonian system pair on $\left(M^{2 k+2 h}, \omega_{1}, \omega_{2}\right), \mu$ the momentum map, $\left(\mathcal{O}_{1}, \mathcal{O}_{2}\right)$ a singular compact orbit pair of rank $(r, s)$. We assume that, the first integrals $f_{r+1}, \cdots, f_{k}$ and $g_{s+1}, \cdots, g_{h}$ have a non-degenerate singularity in the Morse-Bott sense along $\mathcal{O}_{1}$ and $\mathcal{O}_{2}$, respectively. Thus, there exists two triplets of natural numbers $\left(k e_{1}, k h_{1}, k f_{1}\right),\left(k e_{2}, k h_{2}, k f_{2}\right)$ such that $k e_{1}+k h_{1}+2 k f_{1}=k-r$ and $k e_{2}+k h_{2}+2 k f_{2}=h-s$. We recall the notion of linear model. Denote by $\left(x_{1}, \cdots, x_{r}\right)$ a linear coordinate system of a small ball $\mathbb{D}^{r}$ of dimension $r,\left(\alpha_{1}(\bmod 1), \cdots, \alpha_{r}(\bmod 1)\right)$ a standard periodic coordinate system of the torus $\mathbb{T}^{r},\left(y_{1}, z_{1}, \cdots, y_{k-r}, z_{k-r}\right)$ a linear coordinate system of a small ball $\mathbb{D}^{2(k-r)}$ of dimension $2(k-r),\left(p_{1}, \cdots, p_{s}\right)$ a linear coordinate system of a small ball $\mathbb{D}^{s}$ of dimension $s,\left(\beta_{1}(\bmod 1), \cdots, \beta_{s}(\bmod 1)\right)$ a standard periodic coordinate system of the torus $\mathbb{T}^{s},\left(q_{1}, \mu_{1}, \cdots, q_{h-s}, \mu_{h-s}\right)$ a linear coordinate system of a small ball $\mathbb{D}^{2(h-s)}$ of dimension $2(h-s)$. Consider the manifold

$$
M_{0}^{2 k+2 h}=\mathbb{D}^{r} \times \mathbb{T}^{r} \times \mathbb{D}^{2 k-2 r} \times \mathbb{D}^{s} \times \mathbb{T}^{s} \times \mathbb{D}^{2 h-2 s},
$$

with the standard symplectic pair $\omega_{1}^{0}=\sum_{i=1}^{r} d x_{i} \wedge d \alpha_{i}+\sum_{i=1}^{k-r} d y_{i} \wedge d z_{i}$, $\omega_{2}^{0}=\sum_{i=1}^{s} d p_{i} \wedge d \beta_{i}+\sum_{i=1}^{h-s} d p_{i} \wedge d \mu_{i}$ and the following moment map:

$$
\mu_{0}=\left(x_{1}, \cdots, x_{r}, f_{0_{r}+1}, \cdots, f_{0_{k}}, p_{1}, \cdots, p_{s}, g_{0_{s+1}}, \cdots, g_{0_{h}}\right),
$$


where

$$
\begin{aligned}
f_{0_{i+k}} & =y_{i}^{2}+z_{i}^{2}, \text { for, } 1 \leq i \leq k e_{1}, \\
f_{0_{i+k}} & =y_{i} z_{i}, \text { for, } k e_{1}+1 \leq i \leq k e_{1}+k h_{1}, \\
f_{0_{i+k}} & =y_{i} z_{i+1}-y_{i+1} z_{i}, \text { and } \\
f_{0_{i+k+1}} & =y_{i} z_{i}+y_{i+1} z_{i+1}, \text { for } i=k e_{1}+k h_{1}+2 j-1,1 \leq j \leq k f_{1}
\end{aligned}
$$

and

$$
\begin{aligned}
g_{0_{i+h}} & =q_{i}^{2}+\mu_{i}^{2}, \text { for, } 1 \leq i \leq k e_{2}, \\
g_{0_{i+k}} & =q_{i} \mu_{i}, \text { for, } k e_{2}+1 \leq i \leq k e_{2}+k h_{2}, \\
g_{0_{i+k}} & =q_{i} \mu_{i+1}-q_{i+1} \mu_{i}, \text { and } \\
g_{0_{i+k+1}} & =q_{i} \mu_{i}+q_{i+1} \mu_{i+1}, \text { for } i=k e_{2}+k h_{2}+2 j-1,1 \leq j \leq k f_{2} .
\end{aligned}
$$

We denote by $\left(\mathfrak{F}_{1}^{0}, \mathfrak{F}_{2}^{0}\right)$ the linear Lagrangian foliation pair given, respectively by the orbits of the linear distributions $\mathcal{D}_{1}^{0}=<X_{f_{0 i}}, \cdots$, $X_{f_{0 k}}>$ and $\mathcal{D}_{2}^{0}=<X_{g_{0 i}}, \cdots, X_{g_{0 h}}>$, where $X_{f_{0 i}}$ and $X_{g_{0 i}}$ being the Hamiltonian vector fields of $f_{0 i}$ and $g_{0 i}$ in the symplectic pair model $\left(M_{0}^{2 k+2 h}, \omega_{1}^{0}, \omega_{2}^{0}\right)$. Let $\Gamma$ be a group with a symplectic action $\rho(\Gamma)$ on $M_{0}^{2 k+2 h}$, which preserves the moment map $\mu_{0}$. We will say that the action of $\Gamma$ on $M_{0}^{2 k+2 h}$ is linear if it satisfies the following property: $\Gamma$ acts on the product $M_{0}^{2 k+2 h}$ componentwise; the action of $\Gamma$ on $\mathbb{D}^{r}$ and $\mathbb{D}^{s}$ is trivial, its action on $\mathbb{T}^{r}$ and $\mathbb{T}^{s}$ is by translations (with respect to the coordinate system $\left.\left(\alpha_{1}, \cdots, \alpha_{r}\right),\left(\beta_{1}, \cdots, \beta_{s}\right)\right)$ and its action on $\mathbb{D}^{2 k-2 r}$ and $\mathbb{D}^{2 h-2 s}$ is linear (with respect to the coordinate system $\left(y_{1}, z_{1}, \cdots\right.$, $\left.\left.y_{k-r}, z_{k-r}\right),\left(q_{1}, \mu_{1}, \cdots, q_{k-r}, \mu_{k-r}\right)\right)$. Suppose now that $\Gamma$ is a finite 
group with a free symplectic action $\rho(\Gamma)$ on $M_{0}^{2 k+2 h}$, which preserves the moment map and which is linear. Then we can form the quotient symplectic manifold $\widetilde{M}_{0}=M_{0}^{2 k+2 h} / \Gamma$, with an integrable system on it given by the induced moment map as above:

$$
\mu_{0}=\left(x_{1}, \cdots, x_{r}, f_{r+1}, \cdots, f_{k}, p_{1}, \cdots, p_{s}, g_{s+1}, \cdots, g_{h}\right) .
$$

The set pair $\left(\left\{x_{i}=y_{i}=z_{i}=0\right\},\left\{p_{i}=q_{i}=\mu_{i}=0\right\}\right) \subset \widetilde{M}_{0}$ is a compact orbit pair of Williamson type $\left(\left(k e_{1}, k f_{1}, k h_{2}\right),\left(k e_{2}, k f_{2}, k h_{2}\right)\right)$ of the above system. We will call the above system on $\widetilde{M}_{0}$, together with its associated singular Lagrangian foliation, the linear system (or linear model) of Williamson pair type $\left(\left(k e_{1}, k f_{1}, k h_{1}\right),\left(k e_{2}, k f_{2}, k h_{2}\right)\right)$ and twisting group $\Gamma$ (or more precisely, twisting action $\rho(\Gamma)$ ). We will also say that it is a direct model if $\Gamma$ is trivial, and a twisted model if $\Gamma$ is non trivial. Under the above hypotheses, we show the following theorem:

Theorem 4.1. Then there exists a finite group $\Gamma$ and $a$ diffeomorphism taking the Lagrangian foliation pair $\left(\mathfrak{F}_{1}, \mathfrak{F}_{2}\right)$ to the linear Lagrangian foliation pair $\left(\mathfrak{F}_{1}^{0}, \mathfrak{F}_{2}^{0}\right)$ on $M_{0}^{2 h+2 k} / \Gamma$ and taking $\left(\omega_{1}, \omega_{2}\right)$ to $\left(\omega_{1}^{0}, \omega_{2}^{0}\right)$, which sends $\left(\mathcal{O}_{1}, \mathcal{O}_{2}\right)$ to $\left(\mathbb{T}^{r}, \mathbb{T}^{s}\right)$.

Proof. Since the orbits $\mathcal{O}_{1}, \mathcal{O}_{2}$ are compact then the produce $\mathcal{O}_{1} \times \mathcal{O}_{2}$ is a closed of $M^{2 k+2 h}$. The manifold $M^{2 k+2 h}$ being normal, then it exist a local coordinates system $\left(U, \alpha_{1}, \cdots, \alpha_{k}, \beta_{1}, \cdots, \beta_{k}, \theta_{1}, \cdots, \theta_{h}\right.$, $\left.\mu_{1}, \cdots, \mu_{h}\right)$ around $\left(\mathcal{O}_{1} \times \mathcal{O}_{2}\right)$ in $M^{2 k+2 h}$ such that

$$
U \cap\left(\mathcal{O}_{1} \times \mathcal{O}_{2}\right)=\mathcal{O}_{1} \times \mathcal{O}_{2}
$$

Let $N_{1}, N_{2}$ be the submanifolds defined by $N_{1}:\left\{\theta_{1}=\cdots=\theta_{k}=\mu_{1}=\right.$ $\left.\cdots=\mu_{h}=0\right\}$ and $N_{2}:\left\{\alpha_{1}=\cdots=\alpha_{k}=\beta_{1}=\cdots=\beta_{h}=0\right\}$. Observe that 
$\omega_{1}$ and $\omega_{2}$ are respectively, a symplectic forms on $N_{1}$ and $N_{2}$. Thus, the equations

$$
i_{X} \omega_{1}=-d f_{i} \text { and } i_{X} \omega_{2}=-d g_{i}
$$

have a unique well-defined solution when restricted to the symplectic submanifolds $\left(N_{1}, \omega_{1}\right)$ and $\left(N_{2}, \omega_{2}\right)$, respectively. We denote respectively by $Y_{f_{i}}$ and $Z_{g}$ the solution of this equations. With all these information at hand we can write $X_{f_{i}}=Y_{f_{i}}$ and $X_{g_{i}}=Z_{g_{i}}$, where $X_{f_{i}}$ and $X_{g_{i}}$ are the Hamiltonian vector fields with respect to symplectic pair $\left(\omega_{1}, \omega_{2}\right)$. Observe that, the Hamiltonian vector fields $Y_{f_{1}}, \cdots, Y_{f_{k}}$ define a completely integrable Hamiltonian system on $\left(N_{1}, \omega_{1}\right)$ and the Hamiltonian vector fields $Z_{g_{1}}, \cdots, Z_{g_{h}}$ define a completely integrable Hamiltonian system on $\left(N_{2}, \omega_{2}\right)$. Indeed, we have

$$
\begin{aligned}
\left\{f_{i}, f_{j}\right\}_{N_{1}} & =\omega_{1}\left(Y_{f_{i}}, Y_{f_{j}}\right) \\
& =\left(\omega_{1}+\omega_{2}\right)\left(X_{f_{i}}, X_{f_{j}}\right) \\
& =\left\{f_{i}, f_{j}\right\} \\
& =0
\end{aligned}
$$

and

$$
\begin{aligned}
\left\{f_{i}, f_{j}\right\}_{N_{2}} & =\omega_{2}\left(Z_{f_{i}}, Z_{f_{j}}\right) \\
& =\left(\omega_{1}+\omega_{2}\right)\left(X_{f_{i}}, X_{f_{j}}\right) \\
& =\left\{f_{i}, f_{j}\right\} \\
& =0 .
\end{aligned}
$$

Moreover, $\mathcal{O}_{1}$ is a non degenerate compact orbit of rank $r$ of integrable Hamiltonian system $Y_{f_{1}}, \cdots, Y_{f_{k}}$ in $\left(N_{1}, \omega_{1}\right)$ and $\mathcal{O}_{2}$ is a non degenerate 
compact orbit of rank s of integrable Hamiltonian system $Z_{f_{1}}, \cdots, Z_{f_{h}}$ in $\left(N_{2}, \omega_{2}\right)$. According to Eva Miranda and Nguyen Tien Zung theorem (see [13]), there exists a finite group $\Gamma_{1}$ and a diffeomorphism $\phi_{1}$ taking the foliation $\mathfrak{F}_{1}$ to the linear foliation $\mathfrak{F}_{1}^{0}$ on $\left(\mathbb{D}^{r} \times \mathbb{T}^{r} \times \mathbb{D}^{2 k-2 r}\right) / \Gamma_{1}$ and taking $\omega_{1}$ to $\omega_{1}^{0}$ which send $\mathcal{O}_{1}$ to the torus $\mathbb{T}^{r}$. There exists also a finite group $\Gamma_{2}$ and a diffeomorphism $\phi_{2}$ taking the foliation $\mathfrak{F}_{2}$ to the linear foliation $\mathfrak{F}_{2}^{0}$ on $\left(\mathbb{D}^{s} \times \mathbb{T}^{s} \times \mathbb{D}^{2 h-2 s}\right) / \Gamma_{2}$ and taking $\omega_{2}$ to $\omega_{2}^{0}$ which send $\mathcal{O}_{2}$ to the torus $\mathbb{T}^{s}$. Now, we put $\Gamma=\Gamma_{1} \times \Gamma_{2}$ and $\phi=\left(\phi_{1}, \phi_{2}\right), \Gamma$ is a finite group and $\phi$ a diffeomorphism taking the Lagrangian foliation pair $\left(\mathfrak{F}_{1}, \mathfrak{F}_{2}\right)$ to the linear Lagrangian foliation pair $\left(\mathfrak{F}_{1}^{0}, \mathfrak{F}_{2}^{0}\right)$ on $M_{0}^{2 k+2 h} / \Gamma$ and taking $\left(\omega_{1}, \omega_{2}\right)$ to $\left(\omega_{1}^{0}, \omega_{2}^{0}\right)$, which sends $\left(\mathcal{O}_{1}, \mathcal{O}_{2}\right)$ to $\left(\mathbb{T}^{r}, \mathbb{T}^{s}\right)$. This ends the prove of the theorem.

This theorem enables to classify the symplectic pair germs, up to foliation-preserving automorphism, for which the system $\left(f_{1}, \cdots, f_{k}\right.$, $\left.g_{1}, \cdots, f_{h}\right)$ is a completely integrable Hamiltonian system pair in a neighbourhood of a singular compact orbit pair $\left(\mathcal{O}_{1}, \mathcal{O}_{2}\right)$. There is just one class of symplectic pair germs for which the system $\left(f_{1}, \cdots, f_{k}\right.$, $\left.g_{1}, \cdots, g_{h}\right)$, is a completely integrable Hamiltonian system pair.

Theorem 4.2. If $\left(\omega_{1}^{\prime}, \omega_{2}^{\prime}\right)$ is another symplectic pair for which the system $\left(f_{1}, \cdots, f_{k}, g_{1}, \cdots, g_{h}\right)$, is a completely integrable Hamiltonian system pair, then there exists a diffeomorphism $\phi$ defined in a neighbourhood of $\left(\mathcal{O}_{1}, \mathcal{O}_{2}\right)$ such that:

- It fix the orbit pair $\left(\mathcal{O}_{1}, \mathcal{O}_{2}\right)$.

- It preserve the foliation pair $\left(\mathfrak{F}_{1}, \mathfrak{F}_{2}\right)$. 
- $\phi^{*}\left(\omega_{1}^{\prime}\right)=\omega_{1}, \phi^{*}\left(\omega_{2}^{\prime}\right)=\omega_{2}$.

In this case we said that the symplectic pair are equivalent, and we note $\left(\omega_{1}, \omega_{2}\right) \sim_{\left(\mathfrak{F}_{1}, \mathfrak{F}_{2}\right)}\left(\omega_{1}^{\prime}, \omega_{2}^{\prime}\right)$.

\section{References}

[1] G. Bande, Formes de contact généralisé, couples de contact et couples contactosymplectiques, Thése de doctorat, Université de Haute Alsace, 2000.

[2] G. Bande and D. Kotschick, Formes de contact généralisé, couples de contact et couples contacto-symplectiques, Thése de doctorat, Université de Haute Alsace, 2000 .

[3] Y. Colin and San Vu Ngoc, Singular Bohr-Sommerfeld rules for 2D integrable systems, Ann. Scient. Ec. Norm. Sup. 36(2) (2003), 1-55.

[4] C. Curras-Boch and E. Miranda, Symplectic linearisation of singular Lagrangian foliations in $M^{4}$, Differential Geom. Appl. 18(2) (2003), 195-205.

[5] Dufour and P. Molino, Coordonnees actions-angles avec singularites, Preprint, Montpellier, 1984.

[6] L. H. Eliasson, Normal forms for Hamiltonian systems with Poisson commuting integrabls, Commenntarii mathematici Helvetici, Basel (1929), 4-35.

[7] L. H. Eliasson, Hamiltonian Systems with Poisson Commuting Integrabls, PhD. Thesis, 1984.

[8] H. Ito, Action-angle coordinates at singularities for analytic integrable systems, Math. Z. 206(3) (1991), 363-407.

[9] J. Liouville, Note sur l'intégration des équations différentielles de la dynamique, présentée au bureau des longitudes le 29 juin 1853, Journal de Mathématiques Pures et Aappliquées 20 (1855), 137-138.

[10] H. Mineur, Sur les systèmes mécaniques admettant $n$-intégrales premières uniformes et l'extension à ces systèmes de la méthode de quantification de Sommerfeld, C. R. Acad. Sci., Paris 200 (1935), 1571-1573.

[11] H. Mineur, Sur les systèmes mécaniques dans lesquels figurent des paramétres fonctions du temps. Etude des systèmes admettant $n$-intégrales premières uniformes en involution. Extension $\tilde{A}$ ces systèmes des conditions de quantification de BohrSommerfeld, Journal de l'Ecole Polytechnique, III 143 (1937), 173-191; 237-270.

[12] H. Mineur, Reduction des systèmes mécaniques à $\mathrm{n}$ degrés de liberté admettant $n$-intégrales premières uniformes en involution aux systèmes à variable séparées, J. Math Pure Appl. IX 15 (1936), 385-389. 
[13] Eva Miranda and Nguyen Tien Zung, Equivariant normal forms for nondegenerate singular orbits of integrable Hamiltonian systems, preprint 2003,

http://xxx.arxiv.org/abs/math.SG/0302287.

[14] San Vũ Ngoc and Christophe Wacheux, Smooth normal form for integrable Hamiltonian systems near a focus-focus singularity, arXiv:1103:3282v1 [math.SG] 16 Mar 2011.

[15] J. Williamson, On the algebraic problem concerning the normal form of linear dynamical systems, American Journal of Mathematics 58(1) (1936), 141-163. 